\title{
Article \\ Sars-Cov-2 Infection in Patients on Long-Term Treatment with Macrolides in Spain: A National Cross-Sectional Study
}

\author{
Carmen Marina Meseguer Barros ${ }^{1}$, Natalia Alzueta Isturiz ${ }^{2}$, Rita Sainz de Rozas Aparicio ${ }^{3}$, \\ Rafael Aguilella Vizcaíno ${ }^{4}$, Laura López Esteban ${ }^{5,+}$, Sonia Anaya Ordóñez ${ }^{6}$, Itxasne Lekue Alkorta ${ }^{7, \ddagger}$, \\ Salvadora Martín Suances ${ }^{6}$, Jorge Ignacio Jiménez Arce ${ }^{8}$, Maite Fernández Vicente ${ }^{9}$, \\ Yolanda Borrego Izquierdo ${ }^{10}$, Raquel Prieto Sánchez ${ }^{10}$, Silvia Casado Casuso ${ }^{10}$, Rosa Madridejos ${ }^{11}(\mathbb{D}$, \\ Carmen Marquina Verde ${ }^{9}$, , Rosa Tomás Sanz ${ }^{11}$, María Oro Fernández ${ }^{12}$, Sara Gallardo Borge ${ }^{11}$, \\ Eva Lázaro López ${ }^{13}$, María Belén Pina Gadea ${ }^{14}$, Mercedes Pereira Pía ${ }^{15}$, María Victoria Maestre-Sánchez ${ }^{16}$, \\ Esther Ribes-Murillo ${ }^{17}$, Constanza Gómez de Oña ${ }^{18}$, Álvarez María Jesús Lallana ${ }^{14}\left(\mathbb{D}\right.$, Concepción Celaya Lecea ${ }^{19}$, \\ María Ana Prado Prieto ${ }^{20}{ }^{\infty}$, Aranzazu Aranguez Ruiz ${ }^{21}$, Vicente Olmo Quintana ${ }^{22}$, Noemí Villén Romero ${ }^{23}$, \\ Carolina Payá Giner ${ }^{24}$, Angeles Lloret Callejo ${ }^{25}$, Alvaro Fernández Ferreiro ${ }^{26}$, Blanca Basagoiti Carreño ${ }^{27}$, \\ Ana Aurelia Iglesias Iglesias ${ }^{28}$, Antonio Martín Alonso ${ }^{27,} \|$, Ana Díez Alcántara ${ }^{27}$, Esther Marco Tejón ${ }^{29}$, \\ Marta Lestón Vázquez ${ }^{23}, \mathrm{M}^{\text {a }}$ Ángeles Ariza Copado ${ }^{30}$, Marta Aparicio Cueva ${ }^{31}$, Belén Escudero Vilaplana ${ }^{1}$, \\ Marisa Nicieza ${ }^{32}$, Gracia Picazo Sanchiz ${ }^{1}$, Genma María Silva Riádigos ${ }^{1}$, Lucía Jamart Sánchez ${ }^{1}$, \\ Ángel García Álvarez ${ }^{33}$, Antonio García Bonilla ${ }^{34}$, Rafael Herrero Delicado ${ }^{35}$, Virginia Arroyo Pineda ${ }^{36}$, \\ Belén de la Hija ${ }^{36}$, Amelia Troncoso Mariño ${ }^{23}$, Isabel Tofiño González ${ }^{36}{ }^{\complement}$, Mónica Susana Mateu García ${ }^{37}$, \\ Pablo García Vázquez ${ }^{38}$, Joaquín Pérez Martín ${ }^{39}$ and Rocío Fernández-Urrusuno ${ }^{40, *, \mathbb{I} \text { (i) }}$ \\ on behalf of the Infectious Diseases SEFAP Team
}

updates

Citation: Meseguer Barros, C.M.; Alzueta Isturiz, N.; Sainz de Rozas Aparicio, R.; Vizcaíno, R.A.; López Esteban, L.; Anaya Ordóñez, S.; Lekue Alkorta, I.; Martín Suances, S.; Jiménez Arce, J.I.; Fernández Vicente, M.; et al. Sars-Cov-2 Infection in Patients on Long-Term Treatment with Macrolides in Spain: A National Cross-Sectional Study. Antibiotics 2021, 10, 1039. https://doi.org/ 10.3390/antibiotics10091039

Academic Editor: Anna Psaroulaki

Received: 23 July 2021

Accepted: 20 August 2021

Published: 25 August 2021

Publisher's Note: MDPI stays neutral with regard to jurisdictional claims in published maps and institutional affiliations.

Copyright: (C) 2021 by the authors. Licensee MDPI, Basel, Switzerland. This article is an open access article distributed under the terms and conditions of the Creative Commons
1 Service of Pharmacy, Ouest Primary Health Care Area, Madrid Health Service, Madrid C/Alonso Canonum 8, 28933 Madrid, Spain; carmen.meseguer@salud.madrid.org (C.M.M.B.); belenmaria.escudero@salud.madrid.org (B.E.V.); gracia.picazo@salud.madrid.org (G.P.S.); genmamaria.silva@salud.madrid.org (G.M.S.R.); lucia.jamart@salud.madrid.org (L.J.S.)

2 Unit of Drug Assessment, Advice and Research, Navarra Health Service, Pamplona, Plaza de la Paz s/n, 31002 Pamplona, Spain; natalia.alzueta.isturiz@navarra.es

3 Service of Pharmacy, Cruces University Hospital, Osakidetza, Barakaldo, Vizcaya, Plaza Cruces s/n, 48903 Barakaldo, Spain; RITAMARIA.SAINZDEROZASAPARICIO@osakidetza.eus

4 Service of Pharmacy, North Primary Health Care Area, Madrid Health Service, Madrid C/Alfonso Rodriguez Castelao 17, 28034 Madrid, Spain; rafael.aguilella@salud.madrid.org

5 Service of Pharmacy, Center Primary Health Care Area, Madrid Health Service, Madrid C/Alberto López Palacios, 28021 Madrid, Spain; llopeze@salud.madrid.org

6 Service of Pharmacy, Granada Metropolitano Primary Health Care Area, Andalusian Health Service, Complejo Administrativo Almanjayar C/Joaquina Eguaras nº 2, Edificio 2 Planta 1, 18013 Granada, Spain; sonia.anaya.sspa@juntadeandalucia.es (S.A.O.); marias.martin.sspa@juntadeandalucia.es (S.M.S.)

7 Primary Care Pharmacy Service, Integrated Health Organization, Barakaldo-Sestao, Osakidetza, Avenida Antonio Miranda 5, 48902 Barakaldo, Spain; ITXASNE.LECUEALCORTA@osakidetza.eus

8 Clinical Unit Primary Care Pharmacy Area VII Asturias, Principado de Asturias Health Service, Pza. Sindicatos Mineros num 3, 33600 Mieres, Spain; jorgeignacio.jimenez@sespa.es

9 Service of Pharmacy, Burgos Primary Health Care, Castilla y León Health, Burgos, Castilla y León, C/Calle José María de la Puente, 09006 Burgos, Spain; mafernandezvi@saludcastillayleon.es (M.F.V.); cmarquina@saludcastillayleon.es (C.M.V.)

10 Cantabria Primary Care Management, Cantabrian Health Service, C/Vargas nº 577 planta, 39010 Santander, Spain; yolandaborrego77@hotmail.com (Y.B.I.); raquel.prieto@scsalud.es (R.P.S.); silvia.casado@scsalud.es (S.C.C.)

11 Service of Pharmacy, Mutua Terrasa, Barcelona, CAP Sant Cugat, C/de la Mina s/n, 08173 Sant Cugat del Vallès, Spain; rmadridejos@mutuaterrassa.es (R.M.); rtomas@mutuaterrassa.es (R.T.S.); sgallardo@mutuaterrassa.es (S.G.B.)

12 Primary Care Pharmacy and Residential Social Centers, Cantabrian Health Service, Santander, Cantabria, Av Herrera Oria s/n, 39011 Santander, Spain; maria.oro@scsalud.es

13 Clinical Unit Primary Care Pharmacy Area, Area VIII-Asturias, Principado de Asturias Health, Asturias, C/Manuel Suarez s/n, 33930 Langreo, Spain; lazarolopezeva@gmail.com

14 Service of Primary Care Pharmacy, Zaragoza II Sector, Aragón Health Service, Zaragoza, C/Condes de Aragón 30, 50009 Zaragoza, Spain; mbpina@salud.aragon.es (M.B.P.G.); mjlallana@salud.aragon.es (Á.M.J.L.)

15 San Roque Health Center, Lugo Health Area, Cervo Monforte, Rúa Peña Anda 1, 27002 Lugo, Spain; Maria.Mercedes.Pereira.Pia@sergas.es 
Attribution (CC BY) license (https:// creativecommons.org/licenses/by/ $4.0 /)$.
16 Condado-Campiña Health District, La Palma del Condado, C/Ronda de los Legionarios 8, 21700 Huelva, Spain; mariavmaestre@gmail.com

17 Unit of Pharmacy, Lerida Primary Care Direction, Lerida Territorial Management, Catalan Institute of Health, Lerida, Cataluña, Avenida de Blondel 23, 25002 Lleida, Spain; eribes.lleida.ics@gencat.cat

18 Clinical Unit Primary Care Pharmacy Area V, Asturias, Principado de Asturias Health Service, Gijón, Asturias C/Severo Ochoa s/n, 33208 Gijón, Spain; constanzago2016@gmail.com

19 Pharmacy Sub-Directorate, Navarre Health Service, Pamplona, Plaza de la Paz s/n, Planta 4, 31002 Pamplona, Spain; mc.celaya.lecea@navarra.es

20 Service of Pharmacy, Valladolid Primary Health Care, Castilla y Leon Health, Valladolid, Castilla y León, Cardenal Torquemada $n^{\circ}$ 54, 47010 Valladolid, Spain; maprpr@gmail.com

21 Mérida Health Area Management, Merida Hospital, Badajoz, Extremadura, Av. Don Antonio Campos Hoyos 26, 06800 Mérida, Spain; arancha.aranguez@salud-juntaex.es

22 Service of Pharmacy, Gran Canaria Health Area, Canary Islands Health Service, C/Luis Doreste Silva 34-40, Canary Islands Health Service, 35004 Las Palmas de Gran Canaria, Spain; volmqui@gobiernodecanarias.com

23 Primary Care Barcelona City, Catalan Institute of Health, Barcelona, C/Manso 19, $1^{\mathrm{a}}$ Planta, 08015 Barcelona, Spain; nvillenr.bcn.ics@gencat.cat (N.V.R.); mlestonv.bcn.ics@gencat.cat (M.L.V.); atroncoso@gencat.cat (A.T.M.)

24 Health Management Area Campo de Gibraltar Pharmacy Unit, Pharmaceutical Primary Care, Carretera Getares, s/n, 11207 Algeciras, Spain; carolina.paya.sspa@juntadeandalucia.es

25 Primary Care Management, Integrated Care Management Albacete-University Hospital Complex, Castilla-La Mancha Health Service, Albacete, Castilla La Mancha, C/Hermanos Falcó 37, 02006 Albacete, Spain; malloret@sescam.org

26 Directorate of Health Care and Evaluation, Principado de Asturias Health Service, Oviedo, Asturias, Pza del Carbayón 1, 33001 Oviedo, Spain; alvaro.fernandezf@sespa.es

27 Pharmacy Unit, Northwest Primary Health Care Area, Madrid Health Service, Madrid, Avda. De España 7, 28220 Majadahonda, Spain; blanca.basagoiti@salud.madrid.org (B.B.C.); amartina@salud.madrid.org (A.M.A.); adiez@salud.madrid.org (A.D.A.)

28 Pharmacy Service, Regional Hospital of Manacor, Balearic Islands Health Service, Inca, Balearic Islands, Carretera Manacor-Alcudia, s/n,07500 Manacor, Spain; anaaurelia183@hotmail.com

29 Cuenca Primary Care Management, Hospital Virgen de la Luz, Castilla La Mancha Health Service, C/Hermandad de Donantes de Sangre 1, 16002 Cuenca, Spain; emarcot@sescam.jccm.es

30 Pharmacy Unit, Management of Urgencies and Health Emergencies, Murcia, C/Escultor José Sánchez Lozano $N^{\mathrm{o}}$ 7, $2^{\mathrm{a}}$ planta, 30005 Murcia, Spain; mariaa.ariza@carm.es

31 Pharmaceutical Health Area, Health Department Alicante, Valencia C/Pintor Baeza 12, (Edificio gris) $7^{\mathrm{a}}$ planta, 03010 Alicante, Spain; aparicio_marcue@gva.es

32 Principado de Asturias Health Service Pharmacy Coordination, Central Services, Principado de Asturias Health Service, Asturias, Pza Carbayón 1, 33001 Oviedo, Spain; marialuisa.nicieza@sespa.es

33 Pharmacy Service, Tramuntana Sector, Inca Regional Hospital, Balearic Islands Health Service, Balearic Islands, Carretera Vella de Llubí, s/n, 07300 Inca, Spain; angelga@hcin.es

34 Pharmacy Unit, Jerez, Northwest Coast and Sierra de Cádiz Primary Health Care Area, Jerez de la Frontera, Cádiz, C/José Luis Diez n 14, 11402 Jerez de la Frontera, Spain; antonio.garcia.bonilla.sspa@juntadeandalucia.es

35 Pharmaceutical Management Service, General Directorate of Health Assistance, Murciano Health Service,

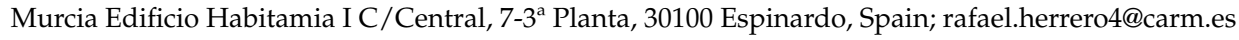

36 Talavera de la Reina Primary Care Management, Nuestra Señora del Prado Hospital, Castilla-La Mancha Health Service, Cuenca, Ctra. Madrid-Extremadura, Km 114, 45600 Toledo, Spain; varroyo@sescam.org (V.A.P.); mbdel@sescam.jccm.es (B.d.1.H.); mitofino@sescam.jccm.es (I.T.G.)

37 Pharmacy Service, Vinarós Department of Health, Castellón, Comunidad Valenciana, Av/Gil de Atrocillo s/n, 12500 Vinaròs, Spain; mateu_mon@gva.es

38 Bierzo Health care Management, El Bierzo Hospital, Castilla y León Health Service, C/Médicos sin Frontera, 7, 24404 Ponferrada, Spain; pgarciav@saludcastillayleon.es

39 Faculty of Social Sciences, Area of Design, Gaming and Multimedia, Madrid, Calle Tajo s/n, 28670 Villaviciosa de Odón, Spain; joaquin.perez@universidadeuropea.es

40 Service of Pharmacy, Aljarafe-Sevilla Norte Primary Health Care Area, Avda de las Américas s/n, 41927 Mairena del Aljarafe, Spain

* Correspondence: rocio.fernandez.sspa@juntadeandalucia.es; Tel.: +34-670945952

+ Current address: Pharmacy Service, Puerta de Hierro University Hospital, C/Joaquín Rodrigo 1, 28222 Majadahonda, Spain.

$\ddagger$ Current address: Primary Care Pharmacy Service, Integrated Health Organization, Barakaldo-Cruces, Osakidetza, Plaza Cruces s/n, 48903 Barakaldo, Spain.

$\S$ Current address: Pharmacy Service, Santos Reyes Hospital, Aranda de Duero, Castilla y León Health, Burgos, Castilla y León, Avda. Ruperta Baraya, 6, 09400 Aranda de Duero, Spain. 
Current address: Emergency Hospital Nurse Isabel Zendal, Av. Manuel Fraga Iribarne 2, 28055 Madrid, Spain.

II Current address: General Directorate of Public Health and Pharmaceutical Management Regional Ministry of Health and Families of Andalusia, Avda. de la Innovación 5, 41020 Sevilla, Spain.

\begin{abstract}
The aim of this study was to know the prevalence and severity of COVID-19 in patients treated with long-term macrolides and to describe the factors associated with worse outcomes. A cross-sectional study was conducted in Primary Care setting. Patients with macrolides dispensed continuously from 1 October 2019 to 31 March 2020, were considered. Main outcome: diagnosis of coronavirus disease-19 (COVID-19). Secondary outcomes: symptoms, severity, characteristics of patients, comorbidities, concomitant treatments. A total of 3057 patients met the inclusion criteria. Median age: 73 (64-81) years; 55\% were men; $62 \%$ smokers/ex-smokers; $56 \%$ obese/overweight. Overall, $95 \%$ of patients had chronic respiratory diseases and four comorbidities as a median. Prevalence of COVID-19: $4.8 \%$. This was in accordance with official data during the first wave of the pandemic. The most common symptoms were respiratory: shortness of breath, cough, and pneumonia. Additionally, $53 \%$ percent of patients had mild/moderate symptoms, $28 \%$ required hospital admission, and 19\% died with COVID-19. The percentage of patients hospitalized and deaths were 2.6 and 5.8 times higher, respectively, in the COVID-19 group $(p<0.001)$. There was no evidence of a beneficial effect of long-term courses of macrolides in preventing SARS-CoV-2 infection or the progression to worse outcomes in old patients with underlying chronic respiratory diseases and a high burden of comorbidity.
\end{abstract}

Keywords: SARS-CoV-2; COVID-19; macrolides; azithromycin; long-term treatment; outpatients; ambulatory care

\title{
1. Introduction
}

The Severe Acute Respiratory Syndrome Coronavirus 2 (SARS-CoV-2) pandemic is having dramatic consequences around the world. Infection prevention is the most desirable solution. Since the beginning of the pandemic, research is under way in order to identify therapies and vaccines for coronavirus disease-19 (COVID-19). Vaccines are being a great success in the fight against this new disease. Their safety and efficacy have been studied in millions of people, although there are still no data on the duration of the protection or on how their efficacy may be influenced by the variants of SARS-CoV-2. However, not all patients may benefit from vaccines, so we cannot rely exclusively on vaccines to end the pandemic. Controlling the COVID-19 pandemic still needs multi-pronged strategies, in addition to effective vaccination and non-pharmacological prophylaxis [1].

The effectiveness of anti-inflammatory agents such as corticoids or tocilizumab and in the effectiveness of antiviral agents suggest that the management of hyperreactivity of the host immune response is advantageous to prevent mortality over targeting viral replication itself $[2,3]$. Macrolides are drugs widely used in the treatment of chronic inflammatory lung diseases to improve pulmonary function based on their antibacterial, antiviral, antiinflammatory, and immunomodulatory properties [4-8]. Long-term macrolides treatments are commonly used in patients with chronic obstructive pulmonary disease (COPD), asthma, bronchiectasis or cystic fibrosis, since they are expected to reduce exacerbations and progression to severe disease [5,6,8-18].

Azithromycin was proposed as a potential therapy for the treatment of SARS-CoV-2 pneumonia at the beginning of the pandemic, due to the publication of a low-quality study with low sample size and many methodological limitations. This study showed that the treatment with hydroxychloroquine in combination with azithromycin had better results in reducing nasal viral load in patients with COVID-19 than hydroxychloroquine in monotherapy [19]. Later, it was ruled out as an option in the treatment of COVID-19 because it did not provide benefit to moderate to severe patients once the disease had progressed 
and required hospitalization. In addition, no effect was observed on mortality, progression to severe course or intensive care unit admission when administered in combination with hydroxychloroquine or with lopinavir-ritonavir, and on time to viral clearance [2,3,20-22]. When used in earlier stages of the disease, azithromycin was not beneficial in patients over 65 years (or over 50 years with at least one comorbidity), in reducing recovery time or decrease the risk of hospitalization [23]. Besides, azithromycin did not reduce the risk of hospitalization or death in patients with mild-moderate COVID-19 managed in the community [24].

These studies provide clear evidence that acute treatment with azithromycin is not an effective treatment for COVID-19 patients after exposure to the virus. However, there are still no results of clinical trials establishing whether long-term treatment with azithromycin or other macrolides could be helpful to prevent disease or worse outcomes. According to the literature, it takes up to 3 months of therapy for macrolides to show a significant effect as immunomodulatory agents and these benefits could disappear 3 months after treatment cessation [6]. Then, this supposed protective effect should be studied in patients who had been receiving macrolides continuously or cyclically for at least 3 months, before exposure to SARS-CoV-2. In clinical practice, patients receiving long courses of macrolides are characterized by their old age, underlying comorbidities and high morbidity and mortality rates [25]. The pandemic is having a higher impact on this population group, with a greater risk of worse outcomes.

The objectives of this work were: (a) to know the prevalence and severity of COVID-19 in adult patients treated with long-term macrolides in Primary Care; (b) to describe the factors that could be associated with worse outcomes.

\section{Results}

\subsection{Study Population}

We identified 3057 patients on long-term treatment with macrolides that met the criteria established in this study.

The baseline characteristics of the study patients are shown in Table 1 . The median age was 73 (64-81) years; $55 \%$ were men; around $62 \%$ were smokers or former smokers, $56 \%$ lived with obesity or overweight $\left(\mathrm{BMI} \geq 25 \mathrm{~kg} / \mathrm{m}^{2}\right)$, and $3 \%$ resided in nursing homes. The great majority of patients (95\%) had chronic respiratory diseases, mainly COPD, bronchiectasis, and asthma. Other common conditions were cardiovascular diseases, neurological/mental, and diabetes mellitus. As a median, they had 4 (3-5) underlying chronic comorbidities, and five risk factors for severe COVID-19.

Table 1. Socio-demographic and baseline clinical characteristics of patients.

\begin{tabular}{|c|c|c|c|c|}
\hline Patient's Characteristics & $\begin{array}{l}\text { Total Patients (n, \%, } \\
\text { Median, } \\
\text { Interquartile Range) }\end{array}$ & $\begin{array}{c}\text { Non-COVID-19 } \\
\text { Patients (n, \%, } \\
\text { Median, Interquartile } \\
\text { Range) }\end{array}$ & $\begin{array}{l}\text { COVID-19 Patients } \\
\text { (n, \%, Median, } \\
\text { Interquartile Range) }\end{array}$ & $p$-Value \\
\hline Total & $3057(100)$ & $2911(100)$ & $146(100)$ & \\
\hline Age (years, median) & $73(64-81)$ & $73(64-81)$ & $74(64-74)$ & 0.164 \\
\hline Sex (n, \%men) & $1687(55.2)$ & $1596(54.8)$ & $91(62.3)$ & 0.049 \\
\hline Smokers or former smokers ${ }^{a}$ & $1611(62.1)$ & $1516(61.5)$ & $95(72.5)$ & 0.010 \\
\hline $\begin{array}{l}\text { Obesity of high body-mass index } \\
\qquad\left(\mathrm{BMI} \geq 25 \mathrm{~kg} / \mathrm{m}^{2}\right)^{\mathrm{b}}\end{array}$ & $1361(55.5)$ & $1297(55.9)$ & $64(49.2)$ & 0.158 \\
\hline $\begin{array}{l}\text { Residence in nursing homes or long-term } \\
\text { care facilities }{ }^{c}\end{array}$ & $98(3.3)$ & $74(2.6)$ & $24(16.4)$ & 0.000 \\
\hline Number of comorbidities, median & $4(3-5)$ & $4(3-5)$ & $4(3-5)$ & 0.904 \\
\hline
\end{tabular}


Table 1. Cont.

\begin{tabular}{|c|c|c|c|c|}
\hline Patient's Characteristics & $\begin{array}{l}\text { Total Patients (n, \%, } \\
\text { Median, } \\
\text { Interquartile Range) }\end{array}$ & $\begin{array}{c}\text { Non-COVID-19 } \\
\text { Patients (n, \%, } \\
\text { Median, Interquartile } \\
\text { Range) }\end{array}$ & $\begin{array}{l}\text { COVID-19 Patients } \\
\text { (n, \%, Median, } \\
\text { Interquartile Range) }\end{array}$ & $p$-Value \\
\hline Respiratory chronic diseases: & $2917(95.4)$ & $2775(95.3)$ & $142(97.3)$ & 0.276 \\
\hline Chronic Obstructive Pulmonary Disease & $1683(55.1)$ & $1596(54.8)$ & $87(59.6)$ & 0.259 \\
\hline Bronchiectasis & $1091(35.7)$ & $1042(35.8)$ & $49(36.6)$ & 0.583 \\
\hline Asthma & $709(23.2)$ & $673(23.1)$ & $36(24.7)$ & 0.667 \\
\hline Chronic respiratory failure & $308(10.1)$ & $286(9.8)$ & $22(15.1)$ & 0.040 \\
\hline Chronic bronchitis & $253(8.3)$ & $241(8.3)$ & $12(8.3)$ & 0.980 \\
\hline Emphysema & $227(7.4)$ & $219(7.5)$ & $8(5.5)$ & 0.358 \\
\hline Lung transplant & $200(6.5)$ & $197(6.8)$ & $3(2.1)$ & 0.025 \\
\hline Cystic fibrosis & $123(4.0)$ & $118(4.1)$ & $5(3.4)$ & 0.706 \\
\hline Other & $527(17.2)$ & $502(17.2)$ & $25(17.2)$ & 0.970 \\
\hline Arterial hypertension & $2001(65.5)$ & $1896(65.1)$ & $105(75.9)$ & 0.092 \\
\hline Cardiovascular, cerebrovascular diseases: & $1231(40.3)$ & $1158(39.8)$ & $73(50.0)$ & 0.014 \\
\hline Cardiovascular disease & $618(20.2)$ & $576(19.8)$ & $42(28.8)$ & 0.008 \\
\hline Heart failure & $408(13.4)$ & $375(13.9)$ & $33(22.6)$ & 0.001 \\
\hline Acute myocardial infarction & $150(4.9)$ & $141(4.8)$ & $9(6.2)$ & 0.471 \\
\hline Stable coronary heart disease & $87(2.9)$ & $82(2.8)$ & $5(3.4)$ & 0.192 \\
\hline Angina pectoris & $59(1.9)$ & $56(1.9)$ & $3(2.1)$ & 0.911 \\
\hline Stroke & $125(4.1)$ & $116(4.0)$ & $9(6.2)$ & 0.194 \\
\hline Peripheral arterial disease & $108(3.5)$ & $100(3.4)$ & $8(5.5)$ & 0.192 \\
\hline Transient ischemic attack & $81(2.7)$ & $76(2.6)$ & $5(3.4)$ & 0.550 \\
\hline Other & $659(21.6)$ & $623(21.4)$ & $36(24.7)$ & 0.351 \\
\hline Chronic neurological or mental diseases: & $964(31.5)$ & $905(31.1)$ & $59(40.4)$ & 0.018 \\
\hline Depression & $566(18.5)$ & $533(18.3)$ & $33(22.6)$ & 0.193 \\
\hline Dementia & $104(3.4)$ & $100(3.4)$ & $4(2.7)$ & 0.651 \\
\hline Parkinson & $42(1.4)$ & $40(1.4)$ & $2(1.4)$ & 0.997 \\
\hline Alzheimer & $31(1.0)$ & $28(1.0)$ & $3(2.1)$ & 0.198 \\
\hline Schizophrenia & $16(0.5)$ & $15(0.5)$ & $1(0.7)$ & 0.782 \\
\hline Other & $355(11.6)$ & $332(11.4)$ & $23(15.8)$ & 0.110 \\
\hline $\begin{array}{l}\text { Situation that leads to } \\
\text { immunosuppression: }\end{array}$ & $753(24.6)$ & $722(24.8)$ & $31(21.2)$ & 0.329 \\
\hline Malignancy & $528(17.3)$ & $504(17.3)$ & $24(16.4)$ & 0.785 \\
\hline Transplant & $174(5.7)$ & $168(5.8)$ & $6(4.1)$ & 0.398 \\
\hline Prolonged use of corticoids & $45(1.5)$ & $42(1.4)$ & $3(2.1)$ & 0.549 \\
\hline Human immunodeficiency virus infection & $20(0.7)$ & $20(0.7)$ & $0(0.0)$ & 0.315 \\
\hline Other & $52(1.7)$ & $48(1.7)$ & $4(2.7)$ & 0.320 \\
\hline Autoimmune disease: & $295(9.7)$ & $284(9.8)$ & $11(7.5)$ & 0.375 \\
\hline Rheumatoid arthritis & $98(3.2)$ & $96(3.3)$ & $2(1.4)$ & 0.197 \\
\hline Psoriasis & $61(2.0)$ & $56(1.9)$ & $5(3.4)$ & 0.206 \\
\hline Inflammatory bowel disease & $34(1.1)$ & $32(1.1)$ & $2(1.2)$ & 0.761 \\
\hline Sjögren Syndrome & $23(0.8)$ & $23(0.8)$ & $0(0.0)$ & 0.281 \\
\hline Lupus erythematosus & $19(0.6)$ & $16(0.6)$ & $3(2.1)$ & 0.024 \\
\hline Celiac disease & $4(0.1)$ & $4(0.1)$ & $0(0.0)$ & 0.110 \\
\hline Multiple sclerosis & $3(0.1)$ & $3(0.1)$ & $0(0.0)$ & 0.698 \\
\hline Other & $82(2.7)$ & $82(2.8)$ & $0(0.0)$ & 0.040 \\
\hline \multicolumn{5}{|l|}{ Other conditions } \\
\hline Diabetes mellitus & $766(25.1)$ & $731(25.1)$ & $35(24.0)$ & 0.757 \\
\hline Chronic kidney failure & $372(12.2)$ & $352(12.1)$ & $20(13.7)$ & 0.562 \\
\hline Liver disease or failure & $137(4.5)$ & $122(4.2)$ & 15 (10.3) & 0.001 \\
\hline Hospital admissions (for any cause) & $684(22.6)$ & $605(21.0)$ & $79(54.5)$ & 0.000 \\
\hline Death & $120(3.9)$ & $93(3.2)$ & $27(18.5)$ & 0.000 \\
\hline
\end{tabular}

a Data from 2596 patients. ${ }^{\mathrm{b}}$ Data from 2452 patients. ${ }^{\mathrm{c}}$ Data from 3014 patients. 
Regarding treatment with macrolides, $98 \%$ of patients received azithromycin, $2 \%$ clarithromycin, and less than $1 \%$ erythromycin. Patients with azithromycin courses have been in treatment for more than 39 months, at a median weekly dose of $1500 \mathrm{mg}$. In addition, as a median, they received 11 concomitant treatments. As shown in Table 2, the most commonly prescribed drugs were bronchodilators, corticoids, proton-pump inhibitors (PPI), antihypertensives, analgesics, benzodiazepines, lipid-lowering agents, and antibiotics (other than macrolides)

Table 2. Treatments with macrolides and concomitant treatments.

\begin{tabular}{|c|c|c|c|c|}
\hline Patient's Treatments & $\begin{array}{l}\text { Total Patients (n, \%, } \\
\text { Median, } \\
\text { Interquartile Range) }\end{array}$ & $\begin{array}{c}\text { Non-COVID-19 } \\
\text { Patients (n, \%, } \\
\text { Median, Interquartile } \\
\text { Range) }\end{array}$ & $\begin{array}{l}\text { COVID-19 Patients } \\
\text { (n, \%, Median, } \\
\text { Interquartile Range) }\end{array}$ & $p$-Value \\
\hline Total & $3057(100)$ & $2911(100)$ & $146(100)$ & \\
\hline Azithromycin & 2987 (97.7) & 2842 (97.6) & $145(99.3)$ & 0.184 \\
\hline Clarithromycin & $55(1.8)$ & $53(1.8)$ & $2(1.4)$ & 0.689 \\
\hline Erythromycin & $16(0.5)$ & $16(0.6)$ & - & - \\
\hline \multicolumn{5}{|l|}{ Days with macrolides ${ }^{a}$, median } \\
\hline Azithromycin & $580(324-1123)$ & $584(327-1124)$ & $468(269-1070)$ & 0.031 \\
\hline Clarithromycin & $354(234-578)$ & $354(231-542)$ & - & - \\
\hline Erythromycin & $456(260-702)$ & $456(260-702)$ & - & - \\
\hline \multicolumn{5}{|l|}{ Weekly dose (mg), median } \\
\hline Azithromycin & $1500(750-1500)$ & $1500(750-1500)$ & $1500(1000-1500)$ & 0.487 \\
\hline Clarithromycin & $7000(3500-7000)$ & $7000(3500-7000)$ & - & - \\
\hline Erythromycin & $2300(1050-3500)$ & $2300(1050-3500)$ & - & - \\
\hline \multicolumn{5}{|l|}{ Current medication } \\
\hline Number of concomitant treatments & $11( \pm 4)$ & $11( \pm 4)$ & $12( \pm 5)$ & 0.000 \\
\hline Bronchodilators: & $2600(85.1)$ & $2467(84.8)$ & $133(91.1)$ & 0.036 \\
\hline Long-acting $\beta 2$-agonist (LABA) & $2279(74.6)$ & $2162(74.3)$ & $117(80.1)$ & 0.112 \\
\hline $\begin{array}{l}\text { Long-acting muscarinic antagonist } \\
\text { (LAMA) }\end{array}$ & $1824(59.7)$ & $1728(59.4)$ & $96(65.8)$ & 0.124 \\
\hline \multirow{2}{*}{$\begin{array}{c}\text { Short-acting } \beta 2 \text {-agonist (SABA) } \\
\text { Short-acting muscarinic antagonist } \\
\text { (SAMA) }\end{array}$} & $1431(46.8)$ & $1361(46.8)$ & $70(47.8)$ & 0.778 \\
\hline & $745(24.4)$ & $705(24.2)$ & $40(27.4)$ & 0.383 \\
\hline Othersystemicantiasthmatics: & $533(17.4)$ & $496(17.1)$ & $37(25.3)$ & 0.010 \\
\hline Montelukast & $390(12.8)$ & $367(12.6)$ & $23(15.8)$ & 0.266 \\
\hline Roflumilast & $127(4.2)$ & $113(3.9)$ & $14(4.2)$ & 0.001 \\
\hline Omalizumab & $10(0.33)$ & $9(0.31)$ & $1(0.68)$ & 0.438 \\
\hline Other & $29(1.0)$ & $27(0.9)$ & $2(1.4)$ & 0.591 \\
\hline Corticoids: & $2491(81.5)$ & $2369(81.4)$ & $122(83.6)$ & 0.508 \\
\hline Inhaled & $2158(70.6)$ & $2045(70.3)$ & $113(77.4)$ & 0.064 \\
\hline Systemic & $870(28.5)$ & $829(28.5)$ & $41(28.1)$ & 0.918 \\
\hline Protonpumpinhibitors & $2247(73.5)$ & $2134(73.3)$ & $113(77.4)$ & 0.275 \\
\hline Antihypertensives & $1919(62.8)$ & $1822(62.6)$ & $97(66.4)$ & 0.348 \\
\hline Analgesics: & $1842(60.3)$ & $1750(60.1)$ & $92(63.0)$ & 0.485 \\
\hline Non-opioids & $1644(53.8)$ & $1560(53.6)$ & $84(57.5)$ & 0.351 \\
\hline Opioids & $660(21.6)$ & $631(21.7)$ & 29 (19.9) & 0.603 \\
\hline Gabapentinoids & $290(9.5)$ & $275(9.5)$ & $15(10.3)$ & 0.739 \\
\hline Benzodiazepines & 1265 (41.4) & $1198(41.2)$ & $67(45.9)$ & 0.257 \\
\hline Lipid-loweringagents & $1225(40.1)$ & $1162(39.9)$ & $63(43.2)$ & 0.437 \\
\hline Antibiotics (otherthanmacrolides) & $893(29.2)$ & $837(28.8)$ & $56(38.4)$ & 0.013 \\
\hline Fluoroquinolones & $387(12.7)$ & $350(12.0)$ & $37(25.3)$ & 0.000 \\
\hline Levofloxacin & $242(7.9)$ & $210(7.2)$ & $32(21.9)$ & 0.000 \\
\hline Ciprofloxacin & $133(4.4)$ & $125(4.3)$ & $8(5.5)$ & 0.493 \\
\hline Moxifloxacin & $58(1.9)$ & $54(1.9)$ & $4(2.7)$ & 0.445 \\
\hline Penicillins & $197(6.4)$ & $188(6.5)$ & $9(6.2)$ & 0.888 \\
\hline
\end{tabular}


Table 2. Cont.

\begin{tabular}{|c|c|c|c|c|}
\hline Patient's Treatments & $\begin{array}{l}\text { Total Patients (n, \%, } \\
\text { Median, } \\
\text { Interquartile Range) }\end{array}$ & $\begin{array}{c}\text { Non-COVID-19 } \\
\text { Patients (n, \%, } \\
\text { Median, Interquartile } \\
\text { Range) }\end{array}$ & $\begin{array}{l}\text { COVID-19 Patients } \\
\text { (n, \%, Median, } \\
\text { Interquartile Range) }\end{array}$ & $p$-Value \\
\hline Amoxicillin-clavulanate & $167(5.5)$ & $160(5.5)$ & $7(4.8)$ & 0.716 \\
\hline Amoxicillin & $35(1.1)$ & $33(1.1)$ & $2(1.2)$ & 0.793 \\
\hline Cephalosporins & $94(3.1)$ & $79(2.7)$ & $15(10.3)$ & 0.000 \\
\hline Cefditoren & $47(1.5)$ & $41(1.4)$ & $6(4.1)$ & 0.010 \\
\hline Cefuroxime & $37(1.2)$ & $35(1.2)$ & $2(1.4)$ & 0.857 \\
\hline Cefixime & $7(0.2)$ & $5(0.2)$ & $2(1.4)$ & 0.003 \\
\hline Ceftriaxone & $7(0.2)$ & $1(0.03)$ & $6(4.1)$ & 0.000 \\
\hline Otherantibiotics & $473(15.5)$ & $452(15.5)$ & $21(14.4)$ & 0.709 \\
\hline Co-trimoxazole & $256(8.4)$ & $247(8.5)$ & $9(6.2)$ & 0.323 \\
\hline Lincosamides-clindamycin & $11(0.4)$ & $10(0.3)$ & $1(0.7)$ & 0.501 \\
\hline Other & $255(8.3)$ & $242(8.3)$ & $13(8.9)$ & 0.801 \\
\hline Antidepressants & $855(28.0)$ & $795(27.3)$ & $60(41.1)$ & 0.000 \\
\hline Antidiabetics & $656(21.5)$ & $625(21.5)$ & $31(21.2)$ & 0.946 \\
\hline Antiplateletdrugs & $638(20.9)$ & $606(20.8)$ & $32(21.9)$ & 0.750 \\
\hline Anticoagulants & $554(18.1)$ & $519(17.8)$ & $35(24.0)$ & 0.060 \\
\hline NSAIDs & $512(16.8)$ & $489(16.8)$ & $23(15.8)$ & 0.741 \\
\hline Antihistamines & $362(11.8)$ & $337(11.6)$ & $25(17.1)$ & 0.043 \\
\hline Immunosuppressants & $321(10.5)$ & $312(10.7)$ & $9(6.2)$ & 0.080 \\
\hline Mucolytics & $306(10.0)$ & $289(9.9)$ & $17(11.6)$ & 0.500 \\
\hline Antipsychotics & $182(6.0)$ & $167(5.7)$ & $15(10.4)$ & 0.024 \\
\hline Antifungals & $165(5.4)$ & $155(5.3)$ & $10(6.9)$ & 0.426 \\
\hline Hydroxychloroquine & $37(1.2)$ & $29(1.0)$ & $8(5.5)$ & 0.000 \\
\hline Coughsuppressants & $27(0.9)$ & $24(0.8)$ & $3(2.1)$ & 0.136 \\
\hline $\begin{array}{l}\text { Number of treatments that increase the } \\
\text { risk of pneumonia } b\end{array}$ & $2(1-4)$ & $2(1-4)$ & $3(2-4)$ & 0.020 \\
\hline
\end{tabular}

${ }^{a}$ Until 31 May2020. ${ }^{b}$ Treatments that increase the risk of pneumonia: antipsychotics, antihistamines, antidepressants, opioids, benzodiazepines, proton-pump inhibitors, immune suppressive agents, gabapentinoids.

A total of $23 \%$ were admitted to the hospital during the study period, and $4 \%$ were dead at the time of data collection.

\subsection{Characteristics of Patients with COVID-19}

A total of 146 cases of COVID-19 were counted: 70 were confirmed cases, 59 were suspected infections, and 17 were probable infections (Table 3). The prevalence of COVID19 was $4.8 \%$ although it widely changed among regions (Table S1, Supplementary data). Most of the cases were detected in March and April. The most common symptoms were respiratory: shortness of breath, cough, and pneumonia. As shown in Table 3, 53\% of patients had mild/moderate symptoms, 28\% required hospital admission, and 19\% died with COVID-19.

Male sex, smokers or ex-smokers, and residents in nursing homes were more represented in the COVID-19 group. There were no differences in the number of comorbidities. However, some conditions including chronic respiratory failure, heart failure, neurological or mental diseases, liver failure, and lupus erythematosus were more prevalent in the COVID-19 group. Lung transplant was less represented among COVID-19 patients (Table 1).

Regarding treatment with macrolides, the great majority of patients (98\%) received azithromycin at a weekly dose of $1500 \mathrm{mg}$ in both groups. There were statistically significant differences in the duration of treatment: COVID-19 patients had been in treatment with macrolides for less time than non-COVID-19 patients $(p<0.031)$ (Table 2$)$. 
Table 3. Clinical characteristics of COVID-19 patients.

\begin{tabular}{|c|c|c|}
\hline Patient's Characteristics & Number of Patients $(n=146, \%)$ & 95\% Confidence Interval \\
\hline $\begin{array}{c}\text { Diagnosis of COVID-19: } \\
\text { Confirmed } \\
\text { Suspected } \\
\text { Probable }\end{array}$ & $\begin{array}{l}70(47.9) \\
59(40.4) \\
17(11.6)\end{array}$ & $\begin{array}{l}(39.6-56.4) \\
(32.4-48.8) \\
(6.9-18.9)\end{array}$ \\
\hline $\begin{array}{l}\text { Date of COVID-19 diagnosis record: } \\
\text { February } \\
\text { March } \\
\text { April } \\
\text { May }\end{array}$ & $\begin{array}{c}2(1.4) \\
53(36.3) \\
58(39.7) \\
33(22.6)\end{array}$ & $\begin{array}{l}(0.2-4.9) \\
(28.5-44.7) \\
(31.7-48.1) \\
(16.1-30.3)\end{array}$ \\
\hline $\begin{array}{l}\text { COVID-19 symptoms: } \\
\text { Asymptomatic } \\
\text { Unspecific * } \\
\text { Respiratory: } \\
\text { Shortness of breath } \\
\text { Cough } \\
\text { Pneumonia } \\
\text { Anosmia, ageusia } \\
\text { Other } \\
\text { Gastrointestinal } \\
\text { Dermatological } \\
\text { Acute kidney failure } \\
\text { Other }\end{array}$ & $\begin{array}{c}13(8.9) \\
5(3.4) \\
121(82.9) \\
86(58.9) \\
63(43.2) \\
41(28.1) \\
5(3.4) \\
8(5.5) \\
17(11.6) \\
3(2.1) \\
2(1.4) \\
7(4.8)\end{array}$ & $\begin{array}{c}(4.8-14.7) \\
(1.1-7.8) \\
(75.8-88.6) \\
(50.5-67.0) \\
(35.0-51.6) \\
(21.0-36.1) \\
(1.1-7.8) \\
(2.4-10.1) \\
(6.9-18.9) \\
(0.4-5.9) \\
(0.2-4.9) \\
(1.9-9.6)\end{array}$ \\
\hline $\begin{array}{c}\text { Severity: } \\
\text { Mild to moderate } \\
\text { Hospitalization with COVID-19 } \\
\text { Death }\end{array}$ & $\begin{array}{l}78(53.4) \\
41(28.1) \\
27(18.5)\end{array}$ & $\begin{array}{l}(45.0-61.7) \\
(21.0-36.1) \\
(12.6-25.8)\end{array}$ \\
\hline
\end{tabular}

* Unspecific: fever, headache, muscle pains, fatigue.

The number of concomitant treatments was higher in the COVID-19 group when compared to the non-COVID-19 group. Drugs more prescribed in COVID-19 patients were bronchodilators, systemic antiasthmatics (roflumilast), antibiotics other than macrolides (third generation cephalosporins and fluoroquinolones), antihistamines, antidepressants, antipsychotics, and hydroxychloroquine. The number of treatments considered to increase the risk of pneumonia was higher in the COVID-19 group $(p=0.002)$. The use of other common drugs, such as corticosteroids, PPI, antihypertensives, or lipid-lowering agents, antiplatelet drugs or anticoagulants did not differ by COVID-19 status.

The percentage of patients admitted to hospital during the study period and the percentage of deaths were 2.6 and 5.8 times higher, respectively, in the COVID-19 group than in the non-COVID-19 group $(p<0.001)$ (Table 1$)$.

\subsection{Factors Associated with Worse Outcomes in COVID-19 Patients}

Patients with severe COVID-19 were older and had more comorbidities than patients with mild/moderate disease (Table 4). Male sex, COPD, bronchiectasis, cardiovascular diseases, diabetes mellitus, and chronic kidney disease were more frequent in patients with worse outcomes although only older age, number of comorbidities, and cardiovascular diseases reached statistical significance. There were no differences in the duration of treatment with macrolides neither in the number of concomitant treatments nor in the type of prescribed drugs between severe and non-severe COVID-19 patients (Table 5).

Regarding recorded symptoms, $27.0 \%$ of patients with cough, $38.4 \%$ with shortness of breath, and $58.5 \%$ with pneumonia were admitted to the hospital. In addition, $14.3 \%$ of patients with cough, $22.1 \%$ with shortness of breath, and $36.6 \%$ with pneumonia died. 
Table 4. Socio-demographic and baseline clinical characteristics of patients with COVID-19 by severity.

\begin{tabular}{|c|c|c|c|c|}
\hline Patient's characteristics & $\begin{array}{l}\text { Total Patients ( } n, \% \text {, } \\
\text { Median, } \\
\text { Interquartile Range) }\end{array}$ & $\begin{array}{l}\text { Mild/Moderate ( } n, \% \text {, } \\
\text { Median, Interquartile } \\
\text { Range) }\end{array}$ & $\begin{array}{c}\text { Hospitalization/Death } \\
(n, \%, \text { Median, } \\
\text { Interquartile Range })\end{array}$ & $p$-Value \\
\hline Total & $146(100)$ & $78(53.4)$ & $68(46.6)$ & \\
\hline Age (years, median) & $74(64-74)$ & $71(63-78)$ & $81(69-86)$ & 0.004 \\
\hline Sex (n, \%men) & $91(62.3)$ & $44(56.4)$ & $47(69.1)$ & 0.114 \\
\hline Smokers or ex-smokers ${ }^{a}$ & $95(72,5)$ & $50(73.5)$ & $45(71.4)$ & 0.788 \\
\hline $\begin{array}{l}\text { Obesity of high body-mass index } \\
\qquad\left(\mathrm{BMI} \geq 25 \mathrm{~kg} / \mathrm{m}^{2}\right)^{\mathrm{b}}\end{array}$ & $64(49.2)$ & $35(51.5)$ & $29(46.8)$ & 0.593 \\
\hline $\begin{array}{l}\text { Residence in nursing homes or long-term } \\
\text { care facilities }\end{array}$ & $24(16.4)$ & $9(11.5)$ & $15(22.1)$ & 0.087 \\
\hline Number of comorbidities, median & $4(3-5)$ & $4(2-5)$ & $5(4-6)$ & 0.014 \\
\hline Respiratory chronic diseases: & $142(97.3)$ & $75(96.1)$ & $67(98.5)$ & 0.380 \\
\hline Chronic Obstructive Pulmonary Disease & $87(59.6)$ & $42(53.8)$ & $45(66.2)$ & 0.130 \\
\hline Bronchiectasis & $49(36.6)$ & $23(29.5)$ & $26(38.2)$ & 0.264 \\
\hline Asthma & $36(24.7)$ & $21(26.9)$ & $15(22.1)$ & 0.496 \\
\hline Chronic respiratory failure & $22(15.1)$ & $11(14.1)$ & $11(16.1)$ & 0.727 \\
\hline Chronic bronchitis & $12(8.3)$ & $8(10.3)$ & $4(5.9)$ & 0.337 \\
\hline Emphysema & $8(5.5)$ & $4(5.1)$ & $4(5.9)$ & 0.842 \\
\hline Other & $30(20.6)$ & $19(24.4)$ & $11(16.2)$ & 0.222 \\
\hline Arterial hypertension & 105 (75.9) & $56(71.8)$ & $49(72.1)$ & 0.972 \\
\hline Cardiovascular, cerebrovascular diseases: & $73(50.0)$ & $33(42.3)$ & $40(58.8)$ & 0.046 \\
\hline Cardiovascular disease & $42(28.8)$ & $22(32.4)$ & $20(25.6)$ & 0.371 \\
\hline Heart failure & $33(22.6)$ & $15(19.2)$ & $18(26.5)$ & 0.297 \\
\hline Acute myocardial infarction & $9(6.2)$ & $4(5.1)$ & $5(7.4)$ & 0.577 \\
\hline Stable coronary heart disease & $5(3.4)$ & $4(5.1)$ & $1(1.5)$ & 0.225 \\
\hline Angina pectoris & $3(2.1)$ & $1(1.3)$ & $2(2.9)$ & 0.481 \\
\hline Stroke & $9(6.2)$ & $6(7.7)$ & $3(4.4)$ & 0.127 \\
\hline Peripheral arterial disease & $8(5.5)$ & $5(6.4)$ & $3(4.4)$ & 0.597 \\
\hline Transient ischemic attack & $5(3.4)$ & $1(1.3)$ & $4(5.9)$ & 0.411 \\
\hline Other & $36(24.7)$ & $14(18.0)$ & $22(32.3)$ & 0.044 \\
\hline Chronic neurological or mental diseases & $59(40.4)$ & $31(39.7)$ & $28(41.2)$ & 0.860 \\
\hline $\begin{array}{l}\text { Situation that leads to } \\
\text { immunosuppression: }\end{array}$ & $31(21.2)$ & $13(16.7)$ & $18(26.5)$ & 0.148 \\
\hline Malignancy & $24(16.4)$ & $11(14.1)$ & $13(19.1)$ & 0.415 \\
\hline Transplant & $6(4.1)$ & $2(2.6)$ & $4(5.9)$ & 0.314 \\
\hline Other & $6(4.1)$ & $1(1.3)$ & $5(7.4)$ & 0.065 \\
\hline Autoimmune disease & $11(7.5)$ & $9(11.5)$ & $2(2.9)$ & 0.050 \\
\hline \multicolumn{5}{|l|}{ Other conditions } \\
\hline Diabetes mellitus & $35(24.0)$ & $15(19.2)$ & $20(29.4)$ & 0.151 \\
\hline Chronic kidney failure & $20(13.7)$ & $7(9.0)$ & $13(19.1)$ & 0.075 \\
\hline Liver disease or failure & $15(10.3)$ & $6(7.7)$ & $9(13.2)$ & 0.271 \\
\hline $\begin{array}{l}\text { Number of severe COVID-19 risk factors }{ }^{c} \text {, } \\
\text { mean }( \pm \text { SD) }\end{array}$ & $6( \pm 2)$ & $5( \pm 2)$ & $6( \pm 2)$ & 0.082 \\
\hline
\end{tabular}

${ }^{a}$ Data from 131 patients. ${ }^{b}$ Data from 130 patients. ${ }^{\mathrm{c}}$ Risk factors for severe COVID-19: $\geq 60$ years, arterial hypertension, cardiovascular disease, diabetes, chronic renal failure, obesity, respiratory chronic disease, malignancy, chronic neurological or mental diseases, liver disease, immune suppression. 
Table 5. Treatments with macrolides and concomitant treatmentsin patients with COVID-19 by severity.

\begin{tabular}{|c|c|c|c|c|}
\hline Patient's Treatment & $\begin{array}{l}\text { Total Patients ( } n, \% \text {, } \\
\text { Median, } \\
\text { Interquartile Range) }\end{array}$ & $\begin{array}{l}\text { Mild/Moderate }(n, \% \text {, } \\
\text { Median, Interquartile } \\
\text { Range) }\end{array}$ & $\begin{array}{l}\text { Hospitalization/Death } \\
(n, \%, \text { Median, } \\
\text { Interquartile Range })\end{array}$ & $p$-Value \\
\hline Total & $146(100)$ & $78(53.4)$ & $68(46.6)$ & \\
\hline \multicolumn{5}{|l|}{$\begin{array}{l}\text { Treatment with long-term macrolides } \\
\text { (azythromycin) }\end{array}$} \\
\hline Number of patients & 145 (99.3) & $77(98.7)$ & $68(100)$ & 0.349 \\
\hline Days with macrolides ${ }^{a}$, median & $468(269-1070)$ & $468(273-936)$ & $499(257-1127)$ & 0.934 \\
\hline Weekly dose (mg), median & $1500(1000-1500)$ & $1500(1000-1500)$ & $1500(1000-1500)$ & 0.584 \\
\hline \multicolumn{5}{|l|}{ Current medication } \\
\hline Number of concomitant treatments & $12( \pm 5)$ & $11( \pm 5)$ & $13( \pm 4)$ & 0.059 \\
\hline Bronchodilators & $133(91.1)$ & $69(88.5)$ & $64(94.1)$ & 0.231 \\
\hline Long-acting $\beta 2$-agonist (LABA) & $117(80.1)$ & $62(79.5)$ & $55(80.9)$ & 0.833 \\
\hline $\begin{array}{l}\text { Long-acting muscarinic antagonist } \\
\text { (LAMA) }\end{array}$ & $96(65.8)$ & $51(65.4)$ & $45(66.2)$ & 0.920 \\
\hline Short-acting $\beta 2$-agonist (SABA) & $70(47.8)$ & $34(43.6)$ & $36(52.9)$ & 0.259 \\
\hline $\begin{array}{l}\text { Short-acting muscarinic antagonist } \\
\text { (SAMA) }\end{array}$ & $40(27.4)$ & $18(23.1)$ & $22(32.4)$ & 0.210 \\
\hline Other systemic antiasthmatics & $37(25.3)$ & $20(25.6)$ & $17(25.0)$ & 0.929 \\
\hline Montelukast & $23(15.8)$ & $13(16.7)$ & $10(14.7)$ & 0.746 \\
\hline Roflumilast & $14(4.2)$ & $7(9.0)$ & $7(10.3)$ & 0.787 \\
\hline Corticoids: & $122(83.6)$ & $63(80.8)$ & $59(86.8)$ & 0.330 \\
\hline Inhaled & $113(77.4)$ & $59(75.6)$ & $54(79.4)$ & 0.587 \\
\hline Systemic & $41(28.1)$ & $18(23.1)$ & $23(33.8)$ & 0.149 \\
\hline Proton pump inhibitors & $113(77.4)$ & $57(73.1)$ & $56(82.4)$ & 0.181 \\
\hline Antihypertensives & $97(66.4)$ & $51(65.4)$ & $46(67.7)$ & 0.773 \\
\hline Analgesics: & $92(63.0)$ & $45(57.7)$ & $47(69.1)$ & 0.154 \\
\hline Non-opioids & $84(57.5)$ & $41(52.6)$ & $43(63.2)$ & 0.193 \\
\hline Opioids & $29(19.9)$ & $17(21.8)$ & $12(17.7)$ & 0.531 \\
\hline Gabapentinoids & $15(10.3)$ & $9(11.5)$ & $6(8.8)$ & 0.590 \\
\hline Benzodiazepines & $67(45.9)$ & $39(50.0)$ & $28(41.2)$ & 0.286 \\
\hline Lipid-loweringagents & $63(43.2)$ & $33(42.3)$ & $30(44.1)$ & 0.826 \\
\hline Antibiotics (other than macrolides) & $56(38.4)$ & $30(38.5)$ & $26(38.2)$ & 0.978 \\
\hline Fluoroquinolones & $37(25.3)$ & $20(25.6)$ & $17(25.0)$ & 0.929 \\
\hline Cephalosporins & $15(10.3)$ & $6(7.7)$ & $9(13.2)$ & 0.271 \\
\hline Penicillins & $9(6.2)$ & $5(6.4)$ & $4(5.9)$ & 0.895 \\
\hline Other antibiotics & $21(14.4)$ & $11(14.1)$ & $10(14.7)$ & 0.917 \\
\hline Antidepressants & $60(41.1)$ & $32(41.0)$ & $28(41.2)$ & 0.985 \\
\hline Antidiabetics & $31(21.2)$ & $12(15.4)$ & $19(27.9)$ & 0.064 \\
\hline Antiplatelet drugs & $32(21.9)$ & $17(21.8)$ & $15(22.1)$ & 0.969 \\
\hline Anticoagulants & $35(24.0)$ & $15(19.2)$ & $20(29.4)$ & 0.151 \\
\hline NSAIDs & $23(15.8)$ & $14(18.0)$ & $9(13.2)$ & 0.435 \\
\hline Antihistamines & $25(17.1)$ & $13(16.7)$ & $12(17.7)$ & 0.875 \\
\hline Immunosuppressants & $9(6.2)$ & $2(2.6)$ & $7(10.3)$ & 0.053 \\
\hline Mucolytics & $17(11.6)$ & $6(7.7)$ & $11(16.2)$ & 0.111 \\
\hline Antipsychotics & $15(10.4)$ & $7(8.8)$ & $8(11.8)$ & 0.580 \\
\hline Antifungals & $10(6.9)$ & $5(6.4)$ & $5(7.4)$ & 0.822 \\
\hline Hydroxychloroquine & $8(5.5)$ & $3(3.9)$ & $5(7.4)$ & 0.353 \\
\hline $\begin{array}{l}\text { Number of treatments that increase the } \\
\text { risk of pneumonia } b\end{array}$ & $3(2-4)$ & $3(2-4)$ & $3(1-4)$ & 0.506 \\
\hline
\end{tabular}

${ }^{a}$ Until 31 May2020. ${ }^{b}$ Treatments that increase the risk of pneumonia: antipsychotics, antihistamines, antidepressants, opioids, benzodiazepines, proton-pump inhibitors, immune suppressive agents, gabapentinoids. 


\section{Discussion}

In this nationwide study, we studied a cohort of patients in treatment with long-term courses of macrolides during the first wave of COVID-19 in Spain. Patients included in this study were characterized by their old age, underlying chronic respiratory diseases, and multiple chronic conditions such as cardiovascular diseases or hypertension, in addition to their respiratory disease. We found a prevalence of COVID-19 infection of $4.8 \%$. This was in accordance with data from the Spanish Ministry of Health, Consumer Affairs and Social Welfare during the first wave of pandemic (5.0-5.2\%) [26], with similar variability between regions and the peak in numbers of people affected in March and April $2020[27,28]$.

Patients with chronic conditions, like those described in this study, deal with colonization, frequent infections and exacerbations, a high rate of Pseudomonas aeruginosa, and multidrug resistant bacteria [25]. These facts make them more susceptible to a poor prognosis and a higher probability of hospitalization and death regardless of COVID-19 [25,29]. The convergence of the threat of COVID-19 and antimicrobial resistance is a cocktail for devastating effects for this population [28,30-38]. Being a very high-risk population, it is supposed that they have probably not left their homes, and have taken strict protective measures against infections: social distancing, the use of face mask and hydroalcoholic disinfectant gel, and strict control of symptoms with medication. This may have helped to prevent exacerbations or worsening of their baseline situation. This would justify the fact that patients in the study who did not get COVID-19, showed a lower degree of hospitalization $(21 \%$ vs. $30 \%)$ and mortality ( $3 \%$ vs. $6 \%$ ) when compared to the same population group studied 3 years before the pandemic in the same setting [25].

Regarding patients who got COVID-19, they were more frequently male, smokers or ex-smokers, and residents in nursing homes. There were no differences in age, number of comorbidities or the presence of chronic respiratory diseases between COVID-19 and non-COVID-19 patients. However, the greater therapeutic burden, the higher use of bronchodilators, other systemic antiasthmatics, and anticoagulants suggest a higher severity in their underlying pathology. It should be noted that patients who acquired COVID-19 had been in treatment with macrolides for less time than those who did not acquire the infection $(p=0.031)$. Given the methodology of the study, we cannot delve further into this observation as further studies are needed to clarify this finding in patients with a longer treatment period with macrolides before SARS-CoV-2 exposition.

The most common reported symptoms in COVID-19 patients were respiratory (shortness of breath, cough, and pneumonia), which are in turn, common in these patients due to their underlying pathology. Despite long-term treatment with macrolides, and the concomitant treatment with other groups of antibiotics in $38 \%$ of patients, pneumonia was reported in $28 \%$ of COVID-19 cases. Pneumonia was the symptom most frequently associated with hospital admissions (58.5\% of patients) and death with COVID-19 (36.6\% of patients).

Antibiotics have shown little therapeutic value in COVID-19, due to the low frequency of bacterial co-infections [39-41]. Their use in the patients in our study would be justified by an attempt to minimize the risk of exacerbations or recurrence of severe bacterial infections that could aggravate the symptoms of COVID-19. However, we did not observe differences in the use of antibiotics (other than macrolides) between patients who progressed to worse outcomes and those who did not. Treatment of high-risk patients with antibiotics in our setting did not have an impact on the prevention of severe COVID-19 outcomes, including pneumonia. Regarding the greater use of hydroxychloroquine, it could be explained by its widespread use during the first months of the pandemic, although there was no clear evidence of benefit [42].

The rate of hospital admissions in COVID-19 patients in our study was lower in comparison with data shown in official national reports (28\% vs. $38-45 \%)[27,28]$. By contrast, case fatality rate was higher $(19 \%$ vs. $8-12 \%)[27,28]$. Mortality is a more reliable outcome to assess severity, since some elderly patients who would have required admission to hospital may not have been admitted because of the high pressure of the healthcare system 
in Spain [28]. When compared to the patients on long-term treatment with macrolides who did not acquire the infection, COVID-19 patients were 3 times more likely to be admitted to hospital and 6 times more likely to die $(p<0.001)$.

According to the available literature $[28,31,34-38,43]$, patients with severe outcomes of COVID-19 were older, male and presented more comorbidities including cardiovascular and cerebrovascular diseases, COPD, bronchiectasis, malignancies, diabetes mellitus, chronic kidney failure and liver failure, and less frequently asthma. However, statistically significant differences were only observed for age, the number of comorbidities and the presence of cardiovascular and cerebrovascular diseases. No statistically significant differences were found in other variables, probably due to the small number of patients.

Given the data, there does not seem to be a different behavior against infection in patients on long-term treatment with macrolides regarding the acquisition of infection or the development of worse outcomes. The morbidity and mortality of our study patients seem to be explained by the same factors as patients who do not receive long-term macrolides treatment.

This study has several strengths. First, it includes a large representative sample of the national territory, including all adult patients cared for by the Public Health Care Services. Full information about the patient's characteristics, underlying pathologies, and medication burden were individually collected. The degree of under-registration of diagnoses in medical records was very low compared to other studies conducted in Primary Care, which reported 30-60\% diagnoses unknown [44].

Second, it is necessary to generate more knowledge in Primary Care. The great majority of the COVID-19 research has been conducted in the hospital setting. More studies are needed in Primary Care, since even for very high-risk patients, such as those included in this study, care/coverage is mostly carried out from this setting (71\% of the study patients were followed up by Primary Care). The use of Primary Care data could help to identify characteristics, evolution risk factors for severe COVID-19 of patients who were not admitted to hospitals.

Third, in contrast with other studies conducted with short-term treatments, the potential anti-inflammatory effect of macrolides (azithromycin) was assessed when administered from long-term before exposure to SARS-CoV-2.

Several limitations of the study should be pointed out. First, it is an observational study. It does not allow us to establish causal associations of the observations. Regarding the control group, there were no data from patients with the same characteristics who have not received long-term macrolides. As an external comparator, we used the Spanish official data. On the other hand, the control group for patients who acquired COVID-19 in the study was the group of patients who did not acquire the disease. Second, patients were included based on medication prescriptions. The ascertainment of medication use from the electronic health record may not reflect real exposure. We had to assume a good therapeutic adherence, based on the pharmacy records of dispensed drugs. Third, we did not have access to hospital records. Information about other symptoms, degree of severity, data on microbiology or treatments may have been under-reported in Primary Care Digital Health History. Fourth, some patients who became symptomatic since the beginning of March were diagnosed based on symptoms because this reflects Primary Care settings, where timely testing might not have been available to all patients particularly at the early stages of the pandemic (testing capacity was limited to patients who needed hospital admission or living in nursing homes). Until mid-April, SARS-CoV-2 PCR or serology diagnostics were not available in Primary Care Health Centers. Even so, $48 \%$ of patients were confirmed cases with molecular diagnostic techniques, a higher value compared to other studies conducted in Primary Care [31]. Fifth, there may be additional confounders influencing outcomes. For example, patients in this study have a high burden of diseases and comorbidities. Multimorbidity and polypharmacy make it challenging to interpret the results, especially in this case, in which patients present chronic respiratory diseases, whose symptoms may be confused with those of COVID-19. 


\section{Materials and Methods}

4.1. Design

A descriptive cross-sectional multicenter study was carried out in the Primary Care setting. The study comprised 47 Healthcare Areas from 16 of the 17 regions in Spain, covering a population of $14,349,076$ people (Table S1, Supplementary data).

Patients $\geq 18$ years of age assigned to Primary Care Centers from the study areas, who had been prescribed and dispensed in community pharmacy at least 10 packages of azithromycin (J01FA10), five packages of clarithromycin (J01FA09) or five packages of erythromycin (J01FA01) for systemic use (according to the WHO Anatomical Therapeutic Chemical classification system) [45], from 1 October 2019 to 31 March 2020 were considered. These agents were selected as the immunomodulatory actions were attributed to 14 - and 15-membered macrolides [6].

The exclusion criteria were: (a) treatment with macrolides started after 31 October 2019; (b) treatment was completed before 29 February 2020; (c) there was any indication that the patient had not received macrolides in a continuous manner, as prescribed; (d) patients without data available in the electronic medical records or those with private pharmacy. The aim was to select patients who were on treatment with macrolides for at least 6 months at the beginning of the pandemic, since it takes up to 3 months of therapy for macrolides to show a supposed significant effect as immunomodulatory agents [6].

\subsection{Data Sources and Outcomes}

Patients were identified through databases from computerized pharmacy records of reimbursed and dispensed drugs, from their Regional Health Care Services. Individual clinical data, diagnoses, and microbiological tests from patients were collected from the electronic medical records of Public Health Services maintained for routine healthcare activities. Population data were obtained from the Statistics National Institute [46].

Data were collected between 15 July 2020 and 30 September 2020. An electronic form, with restricted online access to researchers was designed ad hoc for the data collection. The anonymity of participants was guaranteed by their identification in the electronic form through a numerical code. Data were stored securely in a data center with perimeter security.

The primary outcome was defined as a positive diagnosis of COVID-19.Other outcomes related to COVID-19 disease, were: date of COVID-19 infection diagnosis, symptoms developed: unspecific (fever, headache, muscle pain), respiratory (cough, sore throat, pneumonia, shortness of breath, anosmia, ageusia, odynophagia), dermatological, gastrointestinal, and acute kidney failure. The factors that determined severity were hospitalization and death within the study period.

The data of the final follow-up was 31 May 2020. Patients were classified according to the record that appeared in the Clinical History at the time of data collection. As polymerase chain reaction technique (PCR) and serology diagnostics were not available in the early stages of the pandemic, many patients were diagnosed based on symptoms. The criteria applied for the definition of cases (confirmed: active or past infection; suspected infection; probable infection) were those defined in the latest available protocol of the Spanish Ministry of Health, Consumer Affairs and Social Welfare at the time of the study [47].Due to the continuous adaptation of the protocols regarding the registration of episodes related to COVID-19, the definition of cases in the current protocol at the time of the study is described in Table 6 [47].

The following variables related to demographic and clinical information were collected: age, gender, smoking behavior (current smoking or former smoker), obesity or high body-mass index (BMI) (BMI $\left.>25 \mathrm{~kg} / \mathrm{m}^{2}\right)$, residence in nursing home or in long-term care facilities, the number and type of comorbidities (Table S2, Supplementary data), number of severe COVID-19 risk factors (defined as the sum of: age $\geq 60$ years, arterial hypertension, cardiovascular disease, diabetes, chronic renal failure, obesity, respiratory chronic disease, malignancy, chronic neurological or mental diseases, liver disease, immunosuppression) [48], hospital admissions, death, concomitant therapies (Table S3, Supplementary 
data), and number of treatments that increase the risk of pneumonia (antipsychotics, antihistamines, antidepressants, opioids, benzodiazepines, proton-pump inhibitors, immunosuppressive agents or gabapentinoids) [49].Regarding treatment with macrolides, the following variables were recorded: macrolide agent, days on treatment with macrolides, and average weekly dose.

Table 6. COVID-19 case definition, according to the current protocol at the time of the study [47].

\begin{tabular}{cc}
\hline & Any person with laboratory confirmation of SARS-CoV-2 \\
infection by reverse-transcription PCR (PCR) test (or other \\
molecular diagnostic technique considered appropriate), or \\
patients that meet clinical criteria, with negative PCR or other \\
molecular diagnostic technique considered adequate, and \\
positive result for IgM by serology (not by rapid test).
\end{tabular}

\subsection{Statistical Analysis}

A descriptive analysis of the data was carried out to determine the prevalence and profile of patients. Summary statistics were computed using frequencies and percentages for categorical variables and median (50th percentile), and interquartile range (25-75th percentiles) for continuous variables showing asymmetric distribution. Qualitative variables were expressed as percentages and quantitative variables as mean and standard deviation (SD). Confidence intervals were calculated at $95 \%$ (95\% CI).

A bivariate analysis was performed, followed by the calculation of a hypothesis contrast tests appropriate to the nature of the variables. Comparisons of characteristics among patients were analyzed by the Chi-square test for qualitative variables, except when some of the expected values were under 5 , where the Fisher exact test was applied. For quantitative variables, we used Student's test or U-Mann-Whitney based upon their application criteria.

The level of statistical significance has been set at a $p$-value less than 0.05. STATA Corp. V14 was used for statistical analysis (Stata Corp, College Station, 2015).

\section{Conclusions}

Our findings suggest that there is no beneficial effect of long-term courses of macrolides in preventing SARS-CoV-2 infection or the progression to worse outcomes of COVID-19 in old patients with underlying chronic respiratory diseases and a high burden of comorbidity. Further studies should be performed to confirm these results. Meanwhile, our data can be added to the set of studies showing that the antibacterial effects of azithromycin are unlikely to translate into a significant clinical benefit in COVID-19.

Supplementary Materials: The following are available online at https://www.mdpi.com/article/10 .3390/antibiotics10091039/s1, Table S1: Participating Spanish regions and number of patients provided to study; Table S2: Comorbid conditions analyzed; Table S3: Concomitant treatments analyzed. 
Author Contributions: R.F.-U. and C.M.M.B. designed the project. C.M.M.B. managed the database and conducted the statistical analysis. J.P.M. designed the electronic tool for data collection. R.F.-U. and N.A.I. wrote the manuscript. R.F.-U. is the guarantor. All authors contributed to the collection of the data and provided input to their interpretation. All authors have read and agreed to the published version of the manuscript.

Funding: This study has not received funding.

Institutional Review Board Statement: This research was conducted in accordance with the Declaration of Helsinki and national and institutional legislation in Spain regarding clinical research and personal data protection. It has been approved by Hospital Virgen del Rocío and Virgen Macarena Ethics Committee on Health Research (Seville, Spain) (Code 1226-N-20). The study was classified as EPA-OD (code FIS-MAC-2020-11) by the Spanish Medicines Agency and Health Products (AEMPS).

Informed Consent Statement: Patient consent was waived due to the use of anonymized data, as it was approved by the Ethics Committee.

Data Availability Statement: The data that support the findings of this study are available on request from the corresponding author.

Acknowledgments: The authors would like to thank the members of the Infectious Diseases SEFAP team who collaborated in data collection.

Conflicts of Interest: VO has received funding for conference attendance from Novonordisk Lab and Lilly, and has participated as an author of a book sponsored by Pfizer, and has received a training course enrolment by Amgen Lab. The other authors declare that they have no conflicts of interest to disclose.

\section{References}

1. Flaxman, S.; Mishra, S.; Gandy, A.; Unwin, H.J.T.; Mellan, T.; Coupland, H.; Whittaker, C.; Zhu, H.; Berah, T.; Eaton, J.W.; et al. Estimating the effects of non-pharmaceutical interventions on COVID-19 in Europe. Nature 2020, 584, 257-261. [CrossRef]

2. Kim, M.S.; An, M.H.; Kim, W.J.; Hwang, T.-H. Comparative efficacy and safety of pharmacological interventions for the treatment of COVID-19: A systematic review and network meta-analysis. PLoS Med. 2020, 17, e1003501. [CrossRef]

3. Recovery Collaborative Group. Azithromycin in patients admitted to hospital with COVID-19 (RECOVERY): A randomised, controlled, open-label, platform trial. Lancet 2021, 397, 605-612. [CrossRef]

4. Brusselle, G.G.; Stichele, C.V.; Jordens, P.; Deman, R.; Slabbynck, H.; Ringoet, V.; Verleden, G.; Demedts, I.K.; Verhamme, K.; Delporte, A.; et al. Azithromycin for prevention of exacerbations in severe asthma (AZISAST): A multicentrerandomised double-blind placebo-controlled trial. Thorax 2013, 68, 322-329. [CrossRef]

5. Altenburg, J.; De Graaff, C.S.; Van der Werf, T.S.; Boersma, W.G. Immunomodulatory effects of macrolide antibiotics-part 2: Advantages and disadvantages of long-term, low-dose macrolide therapy. Respiration 2011, 81, 75-87. [CrossRef]

6. Kanoh, S.; Rubin, B.K. Mechanisms of Action and Clinical Application of Macrolides as Immunomodulatory Medications. Clin. Microbiol. Rev. 2010, 23, 590-615. [CrossRef] [PubMed]

7. Porter, J.D.; Watson, J.; Roberts, L.R.; Gill, S.K.; Groves, H.; Dhariwal, J.; Almond, M.H.; Wong, E.; Walton, R.P.; Jones, L.; et al. Identification of novel macrolides with antibacterial, anti-inflammatory and type I and III IFN-augmenting activity in airway epithelium. J. Antimicrob. Chemother. 2016, 71, 2767-2781. [CrossRef]

8. López-Boado, Y.S.; Rubin, B.K. Macrolides as immunomodulatory medications for the therapy of chronic lung diseases. Curr. Opin. Pharmacol. 2008, 8, 286-291. [CrossRef] [PubMed]

9. Zarogoulidis, P.; Papanas, N.; Kioumis, I.; Chatzaki, E.; Maltezos, E. Macrolides: From in vitro anti-inflammatory and immunomodulatory properties to clinical practice in respiratory diseases. Eur. J. Clin. Pharmacol. 2011, 68, 479-503. [CrossRef] [PubMed]

10. Gibson, P.G.; Yang, I.A.; Upham, J.W.; Reynolds, P.N.; Hodge, S.; James, A.L.; Jenkins, C.; Peters, M.J.; Marks, G.B.; Barake, M.; et al. Effect of azithromycin on asthma exacerbations and quality of life in adults with per-sistent uncontrolled asthma (AMAZES): A randomised, double-blind, placebo-controlled trial. Lancet 2017, 390, 659-668. [CrossRef]

11. Hiles, S.A.; McDonald, V.M.; Guilhermino, M.; Brusselle, G.; Gibson, P.G. Does maintenance azithromycin reduce asthma exacerbations? An individual participant data meta-analysis. Eur. Respir. J. 2019, 54, 1901381. [CrossRef]

12. Wong, E.H.C.; Porter, J.D.; Edwards, M.R.; Johnston, S.L. The role of macrolides in asthma: Current evidence and future directions. Lancet Respir. Med. 2014, 2, 657-670. [CrossRef]

13. Herath, S.C.; Normansell, R.; Maisey, S.; Poole, P. Prophylactic antibiotic therapy for chronic obstructive pulmonary disease (COPD). Cochrane Database Syst. Rev. 2018. [CrossRef] [PubMed]

14. Uzun, S.; Djamin, R.S.; Kluytmans, J.A.J.W.; Mulder, P.G.H.; Veer, N.E.V.; Ermens, A.A.M.; Pelle, A.J.; Hoogsteden, H.C.; Aerts, J.G.J.V.; van der Eerden, M.M. Azithromycin maintenance treatment in patients with frequent exacerbations of chronic obstructive 
pulmonary disease (COLUMBUS): A randomised, double-blind, placebo-controlled trial. Lancet Respir. Med. 2014, 2, 361-368. [CrossRef]

15. Wong, C.; Jayaram, L.; Karalus, N.; Eaton, T.; Tong, C.; Hockey, H.; Milne, D.; Fergusson, W.; Tuffery, C.; Sexton, P.; et al. Azithromycin for prevention of exacerbations in non-cystic fibrosis bronchiectasias (EMBRACE): A randomised, double-blind, placebo-controlled trial. Lancet 2012, 380, 660-667. [CrossRef]

16. Altenburg, J.; de Graaff, C.S.; Stienstra, Y.; Sloos, J.H.; van Haren, E.H.; Koppers, R.J.; van der Werf, T.S.; Boersma, W.G. Effect of azithromycin maintenance treatment on infectious exacerbations among patients with non-cystic fibrosis bronchiectasis: The BAT randomized controlled trial. JAMA 2013, 209, 1251-1259. [CrossRef]

17. Southern, K.W.; Barker, P.M.; Solis-Moya, A.; Patel, L. Macrolide antibiotics for cystic fibrosis. Cochrane Database Syst. Rev. 2012, 11, CD002203. [CrossRef]

18. Parnham, M.J.; Haber, V.E.; Giamarellos-Bourboulis, E.J.; Perletti, G.; Verleden, G.M.; Vos, R. Azithromycin: Mechanisms of action an their relevance for clinical applications. Pharmacol. Ther. 2014, 143, 225-245. [CrossRef] [PubMed]

19. Gautret, P.; Lagier, J.-C.; Parola, P.; Hoang, V.T.; Meddeb, L.; Mailhe, M.; Doudier, B.; Courjon, J.; Giordanengo, V.; Vieira, V.E.; et al. Hydroxychloroquine and azithromycin as a treatment of COVID-19: Results of an open-label non-randomized clinical trial. Int. J. Antimicrob. Agents 2020, 56, 105949. [CrossRef]

20. Rosenberg, E.S.; Dufort, E.M.; Udo, T.; Wilberschied, L.A.; Kumar, J.; Tesoriero, J.; Weinberg, P.; Kirkwood, J.; Muse, A.; DeHovitz, J.; et al. Association of Treatment With Hydroxychloroquine or Azithromycin With In-Hospital Mortality in Patients With COVID-19 in New York State. JAMA 2020, 323, 2493-2502. [CrossRef] [PubMed]

21. Cavalcanti, A.B.; Zampieri, F.G.; Rosa, R.G.; Azevedo, L.C.; Veiga, V.C.; Avezum, A.; Damiani, L.P.; Marcadenti, A.; KawanoDourado, L.; Lisboa, T.; et al. Hydroxychloroquine with or without Azithromycin in Mild-to-Moderate Covid-19. N. Engl. J. Med. 2020, 383, 2041-2052. [CrossRef]

22. Furtado, R.H.M.; Berwanger, O.; Fonseca, H.A.; Corrêa, T.D.; Ferraz, L.R.; Lapa, M.G.; Zampieri, F.G.; Veiga, V.C.; Azevedo, L.C.P.; Rosa, R.G.; et al. Azithromycin in addition to standard of care versus standard of care alone in the treatment of patients admitted to the hospital with severe COVID-19 in Brazil (COALITION II): A randomised clinical trial. Lancet 2020, 396, 959-967. [CrossRef]

23. PRINCIPLE Trial Collaborative Group. Azithromycin for community treatment of suspected COVID-19 in people at increased risk of an adverse clinical course in the UK (PRINCIPLE): A randomised, controlled, open-label, adaptative platform trial. Lancet 2021, 397, 1063-1074. [CrossRef]

24. Hinks, T.S.; Cureton, L.; Knight, R.; Wang, A.; Cane, J.L.; Barber, V.S.; Black, J.; Dutton, S.J.; Melhorn, J.; Jabeen, M.F.; et al. A randomised clinical trial of azithromycin versus standard care in ambulatory COVID-19-The ATOMIC2 trial. Trials 2020, 21, 718. [CrossRef] [PubMed]

25. Fernández-Urrusuno, R.; Meseguer Barros, C.M.; Anaya-Ordóñez, S.; Izquierdo, Y.B.; Lallana-Álvarez, M.J.; Madridejos, R.; Tejón, E.M.; Sánchez, R.P.; Rodríguez, O.P.; Gil, M.G.; et al. Patients receiving a high burden of antibiotics in the community in Spain: A cross-sectional study. Pharm. Res. Perspect. 2021, 9, e00692. [CrossRef]

26. Ministerio de Sanidad. Consejo Interterritorial del Sistema Nacional de Salud e Instituto de Salud Carlos III. Estudionacional de Seroepidemiologíade la Infecciónpor SARS-CoV-2 enEspaña (ENE-COVID19). Available online: https:/ /www.mscbs.gob.es / ciudadanos/ene-covid/docs/ESTUDIO_ENE-COVID19_INFORME_FINAL.pdf (accessed on 4 June 2021). (In Spanish)

27. Equipo COVID-19. RENAVE. Informe $\mathrm{n}^{\circ}$ 33. Análisis de Loscasos de COVID-19 Notificados a la RENAVE Hasta el 10 de Mayo enEspaña a 29 de mayo de 2020. Available online: https://www.isciii.es/QueHacemos/Servicios/ VigilanciaSaludPublicaRENAVE/EnfermedadesTransmisibles/Documents/INFORMES/Informes\%20COVID-19/Informe\% 20n\%C2\%BA\%2033.\%20An\%C3\%A1lisis\%20de\%20los\%20casos\%20de\%20COVID-19\%20hasta\%20el\%2010\%20de\%20mayo\% 20en \%20Еspa\%C3\%B1a\%20a\%2029\%20de\%20mayo\%20de\%202020.pdf (accessed on 30 May 2020). (In Spanish)

28. Working Group for the Surveillance and Control of COVID-19 in Spain. The First Wave of the COVID-19 Pandemic in Spain: Characterisation of Cases and Risk Factors for Severe Outcomes, as at 27 April 2020. Available online: https:/ /www.eurosurveillance. org/content/ecdc2020;25:2001431 (accessed on 30 May 2021).

29. Shallcross, L.; Beckley, N.; Rait, G.; Hayward, A.; Petersen, I. Antibiotic prescribing frequency amongst patients in primary care: A cohort study using electronic health records. J. Antimicrob. Chemother. 2017, 72, 1818-1824. [CrossRef]

30. Zhou, F.; Yu, T.; Du, R.; Fan, G.; Liu, Y.; Liu, Z.; Xiang, J.; Wang, Y.; Song, B.; Gu, X.; et al. Clinical course and risk factors for mortality of adult inpatients with COVID-19 in Wuhan, China: A retrospective cohort study. Lancet 2020, 395, $1054-1062$. [CrossRef]

31. Mayer, M.A.; Vidal-Alaball, J.; Puigdellívol-Sánchez, A.; Gomez, F.X.M.; Leis, A.; Peña, J.M. Clinical Characterization of Patients With COVID-19 in Primary Care in Catalonia: Retrospective Observational Study. JMIR Public Health Surveill. 2021,7 , e25452. [CrossRef] [PubMed]

32. Wang, Y.; Chen, J.; Chen, W.; Liu, L.; Dong, M.; Ji, J.; Hu, D.; Zhang, N. Does Asthma Increase the Mortality of Patients with COVID-19?: A Systematic Review and Meta-Analysis. Int. Arch. Allergy Immunol. 2020, 182, 76-82. [CrossRef] [PubMed]

33. Cohen, M.A.; Tavares, J. Who are the Most At-Risk Older Adults in the COVID-19 Era? It's Not Just Those in Nursing Homes. J. Aging Soc. Policy 2020, 32, 380-386. [CrossRef] [PubMed]

34. Ciceri, F.; Castagna, A.; Rovere-Querini, P.; De Cobelli, F.; Ruggeri, A.; Galli, L.; Conte, C.; De Lorenzo, R.; Poli, A.; Ambrosio, A.; et al. Early predictors of clinical outcomes of COVID-19 outbreak in Milan, Italy. Clin. Immunol. 2020, 217, 108509. [CrossRef] 
35. Lee, S.W.; Ha, E.K.; Yeniova, A.Ö.; Moon, S.Y.; Kim, S.Y.; Koh, H.Y.; Yang, J.M.; Jeong, S.J.; Moon, S.J.; Cho, J.Y.; et al. Severe clinical outcomes of COVID-19 associated with proton pump inhibitors: A nation-wide cohort study with propensity score matching. Gut 2021, 70, 76-84. [CrossRef] [PubMed]

36. Hansen, E.S.H.; Moeller, A.L.; Backer, V.; Andersen, M.P.; Kober, L.; Kragholm, K.; Torp-Pedersen, C. Severe outcomes of COVID-19 among patients with COPD and asthma. ERJ Open Res. 2020, 7. [CrossRef]

37. Williamson, E.J.; Walker, A.J.; Bhaskaran, K.; Bacon, S.; Bates, C.; Morton, C.E.; Curtis, H.J.; Mehrkar, A.; Evans, D.; Inglesby, P.; et al. Factors associated with COVID-19-related death using OpenSAFELY. Nature 2020, 584, 430-436. [CrossRef] [PubMed]

38. Reilev, M.; Kristensen, K.B.; Pottegård, A.; Lund, L.C.; Hallas, J.; Ernst, M.T.; Christiansen, C.F.; Sørensen, H.T.; Johansen, N.B.; Brun, N.C.; et al. Characteristics and predictors of hospitalisation and death in the first 11,122 cases with positive RT-PCR test for SARS-CoV-2 in Denmark: A nationwide cohort. Int. J. Epidemiol. 2020, 49, 1468-1481. [CrossRef] [PubMed]

39. Langford, B.J.; So, M.; Raybardhan, S.; Leung, V.; Westwood, D.; MacFadden, D.R.; Soucy, J.-P.R.; Daneman, N. Bacterial co-infection and secondary infection in patients with COVID-19: A living rapid review and meta-analysis. Clin. Microbiol. Infect. 2020, 26, 1622-1629. [CrossRef] [PubMed]

40. Sieswerda, E.; de Boer, M.G.; Bonten, M.M.; Boersma, W.G.; Jonkers, R.E.; Aleva, R.M.; Kullberg, B.-J.; Schouten, J.A.; van de Garde, E.M.; Verheij, T.J.; et al. Recommendations for antibacterial therapy in adults with COVID-19-An evidence based guideline. Clin. Microbiol. Infect. 2020, 27, 61-66. [CrossRef]

41. Oldenburg, C.E.; Pinsky, B.A.; Brogdon, J.; Chen, C.; Ruder, K.; Zhong, L.; Nyatigo, F.; Cook, C.A.; Hinterwirth, A.; Lebas, E.; et al. Effect of Oral Azithromycin vs. Placebo on COVID-19 Symptoms in Outpatients With SARS-CoV-2 Infection: A Randomized Clinical Trial. JAMA 2021, 326, 490-498. [CrossRef]

42. RECOVERY Collaborative Group. Effect of Hydroxychloroquine in Hospitalized Patients with Covid-19. N. Engl. J. Med. 2020, 383, 2030-2040. [CrossRef]

43. Docherty, A.B.; Harrison, E.M.; Green, C.A.; Hardwick, H.E.; Pius, R.; Norman, L.; Holden, K.A.; Read, J.; Dondelinger, F.; Carson, G.; et al. Features of 20133 UK patients in hospital with covid-19 using the ISARIC WHO Clinical Characterisation Protocol: Prospective observational cohort study. BMJ 2020, 369, m1985. [CrossRef]

44. Urrusuno, R.F.; Dorado, M.F.; Arenas, A.V.; Martino, C.S.; Baena, S.C.; Balosa, M.C.M. Improving the appropriateness of antimicrobial use in pri-mary care after the implementation of a local antimicrobial guide in both levels of care. Eur. J. Clin. Pharmacol. 2014, 70, 1011-1020. [CrossRef] [PubMed]

45. WHO Collaborating Centre for Drug Statistics Methodology. ATC/DDD Index 2010. Available online: http://www.whocc.no/ atc_ddd_index (accessed on 5 January 2021).

46. Instituto Nacional de Estadística. Available online: https:/ / www.ine.es (accessed on 4 January 2021).

47. Estrategia de Diagnóstico, Vigilancia y Control en la Fase de Transición de la Pandemia COVID-19. Indicadores de Seguimiento. Updated 12 May 2020. Available online: https:/ /www.mscbs.gob.es/profesionales/saludPublica/ccayes/alertasActual/nCov/ documentos/COVID19_Estrategia_vigilancia_y_control_e_indicadores.pdf (accessed on 4 June 2021). (In Spanish)

48. Ministerio de Sanidad. Enfermedadpor Coronavirus, COVID-19. Updated 17 April 2020. Available online: https://www.mscbs. gob.es/en/profesionales/saludPublica/ccayes/alertasActual/nCov/documentos/20200417_ITCoronavirus.pdf (accessed on 4 June 2021). (In Spanish)

49. Laporte, J.R.; Healey, D. Enmedio de la Pandemiapor SARS-CoV-2, Esnecesariaprudencia con fáRmacos de Consume Frecuente que Incrementan el Riesgo de Neumonía. Dirección General de Productos Sanitarios. Inf Farmacoterde la ComunitatValencian. 2020. Available online: http://www.san.gva.es/documents/152919/8717017/BOLETIN+SARS+1_INFORMAC+ FARMACOTERAP1 (accessed on 4 June 2021). (In Spanish) 